OU-HET-653/2010

\title{
Yukawaon Approach to the Sumino Relation for Charged Lepton Masses
}

\author{
Yoshio Koide \\ Department of Physics, Osaka University, Toyonaka, Osaka 560-0043, Japan \\ E-mail address: koide@het.phys.sci.osaka-u.ac.jp
}

\begin{abstract}
On the basis of a supersymmetric yukawaon model, Sumino's relation for charged lepton masses is re-derived. A relation between values of $K(\mu) \equiv\left(m_{e}+m_{\mu}+m_{\tau}\right) /\left(\sqrt{m_{e}}+\sqrt{m_{\mu}}+\right.$ $\left.\sqrt{m_{\tau}}\right)^{2}$ and $\kappa(\mu) \equiv \sqrt{m_{e} m_{\mu} m_{\tau}} /\left(\sqrt{m_{e}}+\sqrt{m_{\mu}}+\sqrt{m_{\tau}}\right)^{3}$ is investigated without using a relation $K=2 / 3$. Predicted value of $\kappa(\mu)$ is compared with the observed value of $\kappa(\mu)$, and it is concluded that the value $\xi(\mu) \equiv(3 / 2) K(\mu)-1$ is of the order of $10^{-3}$ or less.
\end{abstract}

\section{Introduction}

We know an empirical relation [1]

$$
K \equiv \frac{m_{e}+m_{\mu}+m_{\tau}}{\left(\sqrt{m_{e}}+\sqrt{m_{\mu}}+\sqrt{m_{\tau}}\right)^{2}}=\frac{2}{3}
$$

In conventional mass matrix models, "masses" mean not "pole masses", but "running masses". The relation (1.1) is satisfied with the order of $10^{-5}$ for the pole masses, i.e. $K^{\text {pole }}=(2 / 3) \times$ $(0.999989 \pm 0.000014)$, while it is only valid with the order of $10^{-3}$ for the running masses. This has been a mysterious problem as to the relation (1.1) for long years.

Recently, a possible solution of this problem has been proposed by Sumino [2, 3]: He considers that a flavor symmetry is gauged, and radiative corrections by photon to the charged lepton masses are exactly canceled by those by flavor gauge bosons. Therefore, as far as the charged lepton masses are concerned, the running mass values are exactly equal to the pole mass values. (Hereafter, we will refer to this mechanism as Sumino's mechanism.) Moreover, as a byproduct of his model, he has obtained a charged lepton mass relation [3]

$$
m_{\mu}^{3 / 2}+\sqrt{m_{e} m_{\mu} m_{\tau}}=\sqrt{m_{e} m_{\tau}}\left(\sqrt{m_{e}}+\sqrt{m_{\tau}}\right) .
$$

(We also refer to the relation (1.2) as Sumino's relation on the charged lepton masses.) Then, we can completely determine the charged lepton mass spectrum by using two relations (1.1) and (1.2) simultaneously. As we demonstrate it later on, a predicted value of the electron mass $m_{e}$ is in fairly good agreement with the observed one. The Sumino model is an effective theory with an energy scale $\Lambda \sim 10^{2} \mathrm{TeV}$, so that many visible effects are fruitfully expected in $\mathrm{TeV}$ region physics. Although the topic investigated by Sumino appears to be a narrow and restricted topic, we think that the problems proposed by Sumino will provide a vital clue to new physics. 
In the Sumino model, an effective Yukawa coupling constant $Y_{e}^{\text {eff }}$ in the charged lepton sector is given by $Y_{e}^{e f f} \propto\langle\Phi\rangle\langle\Phi\rangle^{T}$, where $\langle\Phi\rangle$ is a vacuum expectation value (VEV) of a scalar field $\Phi$ with $3 \times 3$ components. Therefore, the quantity $K$ defined in Eq.(1.1) is expressed by

$$
K=\frac{v_{1}^{2}+v_{2}^{2}+v_{3}^{2}}{\left(v_{1}+v_{2}+v_{3}\right)^{2}}
$$

where $\left(v_{1}, v_{2}, v_{3}\right)$ are eigenvalues of $\langle\Phi\rangle$. A derivation of the relation $K=2 / 3$ from a scalar potential model was first tried by the author [4], where $Y_{e}^{e f f} \propto\langle\Phi\rangle\langle\Phi\rangle$ was also assumed. However, in Ref.[4], the field $\Phi$ was assigned to $\mathbf{8}+\mathbf{1}$ of a $\mathrm{U}(3)$ flavor symmetry, so that $Y_{e} \propto \Phi \Phi$ was also assigned to $\mathbf{8}+\mathbf{1}$ of $\mathrm{U}(3)$, while, in the Sumino model, $\Phi \Phi^{T}$ is assigned to 6 of $\mathrm{U}(3)$. In other words, Sumino has assigned the charged lepton fields $e_{L}=\left(e_{1}, e_{2}, e_{3}\right)_{L}$ and $e_{R}=\left(e_{1}, e_{2}, e_{3}\right)_{R}$ to $\mathbf{3}$ and $\mathbf{3}^{*}$ of $\mathrm{U}(3)$, respectively. This assignment is essential for the cancellation between interactions of photon and flavor gauge bosons.

In the derivation of the relation (1.2), Sumino [3] has proposed a scalar potential for $\Phi$

$$
V=\lambda_{1}^{S}\left(v_{1}^{2} v_{3}^{2}+v_{2}^{2} v_{2}^{2}+v_{3}^{2} v_{1}^{2}\right)+\lambda_{2}^{S}\left(v_{1}^{4}+v_{2}^{4}+v_{3}^{4}\right),
$$

where, for convenience, we have expressed his potential in terms of the eigenvalues of $\left(v_{1}, v_{2}, v_{3}\right)$. Under the subsidiary condition $\left(v_{1}^{2}+v_{2}^{2}+v_{3}^{2}\right) /\left(v_{1}+v_{2}+v_{3}\right)^{2}=2 / 3$, minimizing conditions of the potential (1.4) lead to a VEV relation

$$
v_{2}^{3}+v_{1} v_{2} v_{3}-v_{1} v_{3}\left(v_{1}+v_{3}\right)=0
$$

in the limit of $\lambda_{2}^{S} / \lambda_{1}^{S} \rightarrow 0$. The VEV relation (1.5) means the charged lepton mass relation (1.2). (Note that the relation (1.1) is totally symmetric under permutations among $m_{e}, m_{\mu}$ and $m_{\tau}$, while the relation (1.2) is symmetric only under $m_{e} \leftrightarrow m_{\tau}$.) Then, by using the relations (1.1) and (1.2) simultaneously, we can completely determine the charged lepton mass spectrum. It is convenient to express the charged lepton mass spectrum in terms of the following two quantities, $K$ and $\kappa[5]$, where $K$ is defined by Eq.(1.1) and $\kappa$ is defined by

$$
\kappa \equiv \frac{\sqrt{m_{e} m_{\mu} m_{\tau}}}{\left(\sqrt{m_{e}}+\sqrt{m_{\mu}}+\sqrt{m_{\tau}}\right)^{3}}=\frac{v_{1} v_{2} v_{3}}{\left(v_{1}+v_{2}+v_{3}\right)^{3}}
$$

which is sensitive to the value of $m_{e}$. By using Eqs.(1.1) and (1.2), we obtain a predicted value $\kappa=2.20869 \times 10^{-3}$, which is in fairly good agreement with the observed value

$$
\kappa^{\text {pole }}=(2.0633 \pm 0.0001) \times 10^{-3} .
$$

Of course, we can exactly fit a predicted value of $\kappa$ in the Sumino model to the observed value $\kappa^{\text {pole }}$ by adjusting the parameter $\lambda_{2}^{S} / \lambda_{1}^{S}$. However, we would like to notice that the relation (1.2) has already given an almost reasonable value of $m_{e}$ without help of the second term $\left(\lambda_{2}\right.$-term).

We have a great interest in the Sumino model. In this paper, we try to derive the Sumino relation (1.2) on the basis of another approach, i.e. on the basis of the so-called supersymmetric 
Table 1: Energy scale dependence of $\xi(\mu)$ and $\kappa(\mu)$ in cases with $\tan \beta=10$ and $\tan \beta=50$. $\xi$ and $\kappa$ for pole masses are given by $\xi^{\text {pole }}=(0.011 \pm 0.014) \times 10^{-3}$ and $\kappa^{\text {pole }}=(2.0633 \pm 0.0001) \times$ $10^{-3}$. The running mass values of the charged leptons have been quoted from Ref. [8].

\begin{tabular}{|c|c|c|c|c|}
\hline & \multicolumn{2}{|c|}{$\tan \beta=10$} & \multicolumn{2}{c|}{$\tan \beta=50$} \\
\hline Scale & $\xi(\mu)\left[10^{-3}\right]$ & $\kappa(\mu)\left[10^{-3}\right]$ & $\xi(\mu)\left[10^{-3}\right]$ & $\kappa(\mu)\left[10^{-3}\right]$ \\
\hline$\mu=m_{Z}$ & $1.879 \pm+0.002$ & $2.0276 \pm 0.0002$ & $1.879 \pm+0.002$ & $2.0276 \pm 0.0002$ \\
$\mu=10^{3} \mathrm{GeV}$ & $1.95 \pm 0.02$ & $2.0271 \pm 0.0002$ & $2.50 \pm 0.02$ & $2.0219 \pm 0.0002$ \\
$\mu=10^{9} \mathrm{GeV}$ & $2.20 \pm 0.02$ & $2.0247 \pm 0.0002$ & $5.69 \pm 0.02$ & $1.9924 \pm 0.0002$ \\
$\mu=10^{12} \mathrm{GeV}$ & $2.30 \pm 0.02$ & $2.0238 \pm 0.0002$ & $7.03 \pm 0.02$ & $1.9800 \pm 0.0002$ \\
$\mu=2 \times 10^{16} \mathrm{GeV}$ & $2.42 \pm 0.02$ & $2.0227 \pm 0.0002$ & $8.62 \pm 0.02$ & $1.9654 \pm 0.0002$ \\
\hline
\end{tabular}

yukawaon model [6, 7], where the effective coupling constants $Y_{f}^{e f f}(f=u, d, \nu, e)$ in the Yukawa interactions are given by $Y_{f}^{\text {eff }}=\left\langle Y_{f}\right\rangle / \Lambda$ (we call the fields $Y_{f}$ "yukawaons"), and the VEV of $Y_{e}$ is also related to a field $\Phi$ as $\left\langle Y_{e}\right\rangle \propto\langle\Phi\rangle\langle\Phi\rangle$. In the yukawaon model, VEV relations are derived from supersymmetric (SUSY) vacuum conditions, so that such relations are only valid at a high energy scale $\mu \sim \Lambda$. Since the flavor symmetry is completely broken at $\mu \sim \Lambda$, so that the effective coupling constants $Y_{f}^{e f f}$ evolve as those in the standard SUSY model below the scale $\Lambda$. In the yukawaon model, we consider that the value of $K(\mu)$ is not exactly $2 / 3$, but, rather, the value may be somewhat deviated from $K=2 / 3$, i.e.

$$
K(\mu)=\frac{2}{3}(1+\xi(\mu))
$$

with $\xi \sim 10^{-3}$ at $\mu \sim \Lambda$. Similarly, in the yukawaon model, the value of $\kappa(\Lambda)$ does not need to be the observed value (1.7), so that we can regard the predicted value $\kappa=2.209 \times 10^{-3}$ from the Sumino relation (1.2) as a value of $\kappa(\mu)$ at $\mu \sim \Lambda$ in the yukawaon model. For a reference, we list values of $\xi(\mu)$ and $\kappa(\mu)$ in Table 1 . The running mass values used in Table 1 have been quoted from Ref. [8]. Also, in Fig.1, we plot those values as a $\xi-\kappa$ curve. The data points from the left to the right in Fig.1 correspond to $\xi-\kappa$ values at $\mu=m_{Z}, 10^{3} \mathrm{GeV}, 10^{9} \mathrm{GeV}, 10^{12} \mathrm{GeV}$ and $2 \times 10^{16} \mathrm{GeV}$, respectively. The "observed" $\xi-\kappa$ curve is dependent on the value of $\tan \beta$ in the estimates of the running charged lepton masses. (Hereafter, we refer to the values of $\xi(\mu)$ and $\kappa(\mu)$ which are calculated by the running mass values $m_{e i}(\mu)$ estimated from the observed (pole) masses $m_{e i}$ as "observed" values of $\xi(\mu)$ and $\kappa(\mu)$ in contrast to the "predicted" values of $\xi(\mu)$ and $\kappa(\mu)$ in the present model.)

As in seen in in Fig.1, it is worthwhile noticing that both $\xi-\kappa$ curves for $\tan \beta=10$ and $\tan \beta=50$ are almost plotted on a common linear curve. We can confirm that the deviation from the common linear relation is of the order of $10^{-2}$ or less. Therefore, the relation given in Fig. 1 is useful to compare our prediction values of $\xi(\mu)-\kappa(\mu)$ with the observed values without assuming a specific value of $\tan \beta$.

In the Sumino model, the subsidiary condition $\left(v_{1}^{2}+v_{2}^{2}+v_{3}^{2}\right) /\left(v_{1}+v_{2}+v_{3}\right)^{2}=2 / 3$ [i.e. the relation (1.1)] was indispensable in deriving the Sumino relation (1.5). In the present paper, we 
try to derive the Sumino relation (1.2) [or (1.5)] without assuming the relation (1.1), i.e. we derive the Sumino relation which is valid even when the relation (1.1) is not satisfied. Although, for the charged lepton mass spectra, some of ideas [9, 7, 5] have already been proposed on the basis of a yukawaon model, we do not adopt such a model in the present paper. Therefore, the present yukawaon model does not predict a value of $K(\Lambda)=(2 / 3)(1+\xi(\Lambda))$, but we can give only a relation between values $\xi(\mu)$ and $\kappa(\mu)$ numerically. We will speculate a possible value $\xi(\Lambda)$ from the observed value of $\kappa(\Lambda)$.

In Sec.2, we give a supersymmetric yukawaon model in the charged lepton sector. A VEV form $\langle\Phi\rangle$ in the present model takes a diagonal form $\langle\Phi\rangle=\operatorname{diag}\left(v_{1}, v_{2}, v_{3}\right)$ in contrast to $\langle\Phi\rangle$ in the Sumino model in which $\langle\Phi\rangle$ cannot be diagonal although $\langle\Phi\rangle\langle\Phi\rangle^{T}$ is diagonal. Therefore, the present model is very intuitive, but it needs many additional fields concerned with mass relations, so that it will somewhat be complicated rather than the Sumino model. The present model will contain two parameters. One is $\xi(\mu)$ defined in Eq.(1.8), because we do not consider $K=2 / 3$. Another one is a parameter $\rho$ which is given by a ratio of coefficients of the superpotential terms. In the limit of $\xi \rightarrow 0$ and $\rho \rightarrow 0$, the result becomes the Sumino mass relation (1.2). In Sec.3, in order to compare our results to the observed values given in Table 1, we will speculate that the value of $\rho$ is given by $\rho=3 / 2$. Then, we can completely determine the value of $\kappa(\mu)$ as a function of $\xi(\mu)$. We will find that two options are possible: one is to accept the Sumino mechanism, because a predicted value of $\kappa$ at $\xi=0$ is in favor of the observed value of $\kappa^{\text {pole }}$, and another one is to consider that the energy scale $\Lambda$ of the effective theory is $\Lambda \sim 10^{4} \mathrm{GeV}$, because our result shows $\kappa(\mu)^{\text {pred }}=\kappa(\mu)^{\text {obs }}$ at $\mu \sim 10^{4} \mathrm{GeV}$. In Sec.2, we assume ad hoc forms of VEV matrices for some specific fields, for example, $\langle E\rangle=v_{E} \mathbf{1}$, and so on. In Sec.4, we will discuss a possible scenario which provides such specific forms. Finally, Sec.5 is devoted to conclusions and remarks.

\section{Model}

Stimulated by the Sumino model, in this paper, we assign the yukawaon $Y_{e}$ to $\mathbf{6}$ of $\mathrm{U}(3)$, not to $\mathbf{8}+\mathbf{1}$ of $\mathrm{U}(3)$ [9] (and also not to $\mathbf{5}+\mathbf{1}$ of $\mathrm{O}(3)$ [10]), so that the would-be Yukawa interaction in the charged lepton sector is given by

$$
H_{Y u k a w a}=\frac{y_{e}}{\Lambda} \sum_{i, j} \ell^{i}\left(Y_{e}\right)_{i j}\left(e^{c}\right)^{j} H_{d},
$$

where $\ell=\left(\nu_{L}, e_{L}\right)$ and $e^{c}$ are $\mathrm{SU}(2)_{L}$ doublet and singlet fields of the leptons, respectively, and $H_{d}$ is the down-type $\mathrm{SU}(2)_{L}$ doublet Higgs scalar in the conventional model. We list fields which are concerned with the charged lepton masses in Table 2. In order to distinguish each yukawaon $Y_{f}$ from others, we assume a $\mathrm{U}(1)_{X}$ symmetry, and we assign a $\mathrm{U}(1)_{X}$ charge ("sector" charge) $Q_{X}$ as $Q_{X}\left(Y_{f}\right)=x_{f}, Q_{X}\left(f^{c}\right)=-x_{f}$, and $Q_{X}=0$ for all $\mathrm{SU}(2)_{L}$ doublet fields. However, in this paper, since we deal with only fields concerned with $Y_{e}$, we denote $Q_{X}\left(Y_{e}\right)=x_{e}$ as $Q_{X}\left(Y_{e}\right)=+1$ simply. (Also, hereafter, we denote $Y_{e}$ as $Y$ simply.) In the $R$ charge assignments in Table 2, the values of $r_{1}$ and $r_{2}$ must be chosen as the charges of $Y, E$ and $\Theta_{T}$ (and also those of $\bar{Y}, \bar{E}$ and $\bar{T}$ ) are different among them, and as an unwelcome combination $E \bar{T} E \bar{T}$ is forbidden. At present, we do not specify those values $r_{1}$ and $r_{2}$. The $\mathrm{Z}_{2}$ parity in the Table 2 has been introduced for 
Table 2: Quantum numbers of fields concerned with the charged lepton masses

\begin{tabular}{|c|cccccccc|}
\hline Field & $Y$ & $E$ & $\Theta_{T}$ & $\bar{Y}$ & $\bar{E}$ & $\bar{T}$ & $\Phi$ & $\tilde{\Phi}$ \\
\hline $\mathrm{U}(3)$ & $\mathbf{6}$ & $\mathbf{6}$ & $\mathbf{6}$ & $\mathbf{6}^{*}$ & $\mathbf{6}^{*}$ & $\mathbf{6}^{*}$ & $\mathbf{8}+\mathbf{1}$ & $\mathbf{8}+\mathbf{1}$ \\
$Q_{X}$ & 1 & 1 & 1 & -1 & -1 & -1 & 0 & 0 \\
$Q_{R}$ & 0 & $r_{1}$ & $r_{1}+2 r_{2}$ & $2-r_{1}-2 r_{2}$ & $2 r_{2}$ & $1-\frac{1}{2} r_{1}-r_{2}$ & $r_{2}$ & $1-r_{2}$ \\
$\mathrm{Z}_{2}$ & + & + & + & + & + & - & + & + \\
\hline
\end{tabular}

the purpose to forbidden a term which includes $\bar{T}$ of an odd number such as $\operatorname{Tr}[\Phi \tilde{\Phi} E \bar{T}]$.

According to the quantum numbers given in Table 2, a possible superpotential $W$ is given as follows: $W=W_{Y}+W_{T}+W_{S}$,

$$
\begin{gathered}
W_{T}=\mu_{T} \operatorname{Tr}\left[\Theta_{T} \bar{Y}\right]+\frac{\lambda_{T}}{\Lambda} \operatorname{Tr}\left[\Theta_{T} \bar{T} Y \bar{T}\right] \\
W_{Y}=\frac{\lambda_{E}}{\Lambda} \operatorname{Tr}[Y \bar{E} E \bar{Y}]+\frac{\lambda_{Y}}{\Lambda} \operatorname{Tr}[\tilde{\Phi} \tilde{\Phi} Y \bar{E}]+\frac{\bar{\lambda}_{Y}}{\Lambda} \operatorname{Tr}[\Phi \Phi E \bar{Y}] \\
W_{S}=\frac{\lambda_{1}}{\Lambda} \operatorname{Tr}[\Phi \Phi \tilde{\Phi} \tilde{\Phi}]+\frac{\lambda_{1}^{\prime}}{\Lambda} \operatorname{Tr}[\Phi \tilde{\Phi} \Phi \tilde{\Phi}]+\frac{\lambda_{2}}{\Lambda} \operatorname{Tr}[\Phi \Phi] \operatorname{Tr}[\tilde{\Phi} \tilde{\Phi}]+\frac{\lambda_{2}^{\prime}}{\Lambda} \operatorname{Tr}[\Phi \tilde{\Phi}] \operatorname{Tr}[\Phi \tilde{\Phi}]+\frac{\lambda_{3}}{\Lambda} \operatorname{Tr}^{2}[\Phi] \operatorname{Tr}^{2}[\tilde{\Phi}] .
\end{gathered}
$$

Here, the potential $W_{T}$ plays a role in giving a relation between $Y$ and $\bar{Y}$. The potential $W_{Y}$ plays a role in providing VEV relations $Y \propto \Phi E \Phi^{T}$ and so on. Since $\Phi$ and $\tilde{\Phi}$ are $\mathbf{8}+\mathbf{1}$ of $\mathrm{U}(3)$, we can consider terms $\operatorname{Tr}\left[\tilde{\Phi} Y \tilde{\Phi}^{T} \bar{E}\right]$ and $\operatorname{Tr}\left[Y \tilde{\Phi}^{T} \tilde{\Phi}^{T} \bar{E}\right]$ in addition to the term $\operatorname{Tr}[\tilde{\Phi} \tilde{\Phi} Y \bar{E}]$. However, since $\langle\tilde{\Phi}\rangle,\langle Y\rangle$ and $\langle\bar{E}\rangle$ can take diagonal forms simultaneously, those terms give the same contributions. Therefore, for simplicity, we have denoted only the term $\operatorname{Tr}[\tilde{\Phi} \tilde{\Phi} Y \bar{E}]$ as a typical one of those in the expression (2.3). (For the $\bar{\lambda}_{Y}$ term, the situation is similar to the $\lambda_{Y}$ term.) The potential $W_{S}$ plays a role in deriving the Sumino relation. Only in the potential $W_{S}$, we have assumed terms of new types $\operatorname{Tr}[A B] \operatorname{Tr}[C D]$ and $\operatorname{Tr}[A] \operatorname{Tr}[B] \operatorname{Tr}[C] \operatorname{Tr}[D]$ in addition to a type $\operatorname{Tr}[A B C D]$. Since we have considered that those terms are written up via two steps, a replacement $\operatorname{Tr}[A B C D] \rightarrow \operatorname{Tr}[A B] \operatorname{Tr}[C D]$ and simultaneous replacements $\operatorname{Tr}[A B] \rightarrow \operatorname{Tr}[A] \operatorname{Tr}[B]$ and $\operatorname{Tr}[C D] \rightarrow \operatorname{Tr}[C] \operatorname{Tr}[D]$, we did not consider such terms $\operatorname{Tr}[A B C] \operatorname{Tr}[D], \operatorname{Tr}[A B] \operatorname{Tr}[C] \operatorname{Tr}[D]$, and so on.

From the superpotential $W=W_{Y}+W_{T}+W_{S}$, we can obtain the following VEV relations. From a SUSY vacuum condition $\partial W / \partial \Theta_{T}=0$, we obtain a VEV relation

$$
\bar{Y}=-\frac{\lambda_{T}}{\mu_{T} \Lambda} \bar{T} Y \bar{T} .
$$

A condition $\partial W / \partial \bar{T}=0$ leads to

$$
\frac{\partial W}{\partial \bar{T}}=\frac{\lambda_{T}}{\Lambda}\left(Y \bar{T} \Theta_{T}+\Theta_{T} \bar{T} Y\right)=0 .
$$


Since we want $\langle Y\rangle \neq 0$ and $\langle\bar{T}\rangle \neq 0$, we choose a vacuum with $\left\langle\Theta_{T}\right\rangle=0$. From SUSY vacuum conditions $\partial W / \partial \bar{Y}=0$ and $\partial W / \partial Y=0$, we obtain

$$
Y \bar{E}=-\frac{\bar{\lambda}_{Y}}{\lambda_{E}} \Phi \Phi, \quad E \bar{Y}=-\frac{\lambda_{Y}}{\lambda_{E}} \tilde{\Phi} \tilde{\Phi}
$$

respectively, where we have used $\left\langle\Theta_{T}\right\rangle=0$. Then, we can see that $\partial W / \partial E=0$ and $\partial W / \partial \bar{E}=0$ are automatically satisfied under the conditions (2.7). For the moment, we assume the following VEV forms for $E, \bar{E}$ and $\bar{T}$ :

$$
\frac{1}{v_{E}}\langle E\rangle=\frac{1}{\bar{v}_{E}}\langle\bar{E}\rangle=\left(\begin{array}{ccc}
1 & 0 & 0 \\
0 & 1 & 0 \\
0 & 0 & 1
\end{array}\right), \quad \frac{1}{\bar{v}_{T}}\langle\bar{T}\rangle=\left(\begin{array}{ccc}
0 & 0 & 1 \\
0 & 1 & 0 \\
1 & 0 & 0
\end{array}\right),
$$

on the basis in which $\langle\Phi\rangle$ takes a diagonal form $\langle\Phi\rangle=\operatorname{diag}\left(v_{1}, v_{2}, v_{3}\right) \equiv v_{0} \operatorname{diag}\left(z_{1}, z_{2}, z_{3}\right)$. (The forms of $\langle E\rangle$ and $\langle\bar{T}\rangle$ will be discussed in Sec.4.) Therefore, the forms $\langle Y\rangle,\langle\bar{Y}\rangle$ and $\langle\bar{\Phi}\rangle$ are expressed as $\langle Y\rangle=v_{Y} Z^{2},\langle\bar{Y}\rangle=\bar{v}_{Y} \bar{Z}^{2}$ and $\langle\tilde{\Phi}\rangle=\bar{v}_{0} \bar{Z}$, where

$$
Z=\operatorname{diag}\left(z_{1}, z_{2}, z_{3}\right), \quad \bar{Z}=\operatorname{diag}\left(z_{3}, z_{2}, z_{1}\right),
$$

$v_{Y} \bar{v}_{E}=-\left(\bar{\lambda}_{Y} / \lambda_{E}\right) v_{0}^{2}, \bar{v}_{Y} v_{E}=-\left(\lambda_{Y} / \lambda_{E}\right) \bar{v}_{0}^{2}$ and $\bar{v}_{Y}=-\left(\lambda_{T} / \mu_{T} \Lambda\right) v_{Y}$.

The Sumino relation is obtained as follows. From a condition $\partial W / \partial \Phi=0$, we obtain

$$
\begin{gathered}
\frac{\partial W}{\partial \Phi}=\frac{\bar{\lambda}_{Y}}{\Lambda}\left(\Phi E \bar{Y}+E \bar{Y} \Phi^{T}\right)+\frac{\lambda_{1}}{\Lambda}(\Phi \tilde{\Phi} \tilde{\Phi}+\tilde{\Phi} \tilde{\Phi} \Phi)+2 \frac{\lambda_{1}^{\prime}}{\Lambda} \tilde{\Phi} \Phi \tilde{\Phi} \\
+2 \frac{\lambda_{2}}{\Lambda} \operatorname{Tr}[\tilde{\Phi} \tilde{\Phi}] \Phi+2 \frac{\lambda_{2}^{\prime}}{\Lambda} \operatorname{Tr}[\Phi \tilde{\Phi}] \tilde{\Phi}+2 \frac{\lambda_{3}}{\Lambda} \operatorname{Tr}^{2}[\Phi] \operatorname{Tr}^{2}[\tilde{\Phi}] \mathbf{1}=0
\end{gathered}
$$

which leads to an equation for the parameters $z_{i}$

$$
c_{0} \mathbf{1}+c_{1} Z+c_{1}^{\prime} \bar{Z}+c_{3} Z \bar{Z}^{2}=0,
$$

where $c_{0}=\lambda_{3} v_{0} \bar{v}_{0}^{2}\left(z_{1}+z_{2}+z_{3}\right)\left(z_{1}^{2}+z_{2}^{2}+z_{3}^{2}\right), c_{1}=\lambda_{2} v_{0} \bar{v}_{0}^{2}\left(z_{1}^{2}+z_{2}^{2}+z_{3}^{2}\right), c_{1}^{\prime}=\lambda_{2}^{\prime} v_{0} \bar{v}_{0}^{2}\left(z_{2}^{2}+2 z_{1} z_{3}\right)$, and $c_{3}=\bar{\lambda}_{Y} v_{E} \bar{v}_{Y} v_{0}+\left(\lambda_{1}+\lambda_{1}^{\prime}\right) v_{0} \bar{v}_{0}^{2}$. Also, from $\partial W / \partial \tilde{\Phi}=0$, we obtain

$$
\bar{c}_{0} \mathbf{1}+\bar{c}_{1} \bar{Z}+\bar{c}_{1}^{\prime} Z+\bar{c}_{3} \bar{Z} Z^{2}=0,
$$

where $\bar{c}_{0}=\lambda_{3} \bar{v}_{0} v_{0}^{2}\left(z_{1}+z_{2}+z_{3}\right)\left(z_{1}^{2}+z_{2}^{2}+z_{3}^{2}\right), \bar{c}_{1}=\lambda_{2} \bar{v}_{0} v_{0}^{2}\left(z_{1}^{2}+z_{2}^{2}+z_{3}^{2}\right), \bar{c}_{1}^{\prime}=\lambda_{2}^{\prime} \bar{v}_{0} v_{0}^{2}\left(z_{2}^{2}+2 z_{1} z_{3}\right)$ and $\bar{c}_{3}=\lambda_{Y} \bar{v}_{E} v_{Y} \bar{v}_{0}+\left(\lambda_{1}+\lambda_{1}^{\prime}\right) \bar{v}_{0} v_{0}^{2}$. If we assume that $W_{Y}$ is symmetric under $\Phi \leftrightarrow \tilde{\Phi}, Y \leftrightarrow \bar{Y}$ and $E \leftrightarrow \bar{E}$, so that $\bar{v}_{0}=v_{0}, \bar{v}_{Y}=v_{Y}$ and $\bar{v}_{E}=v_{E}$, we find that Eq.(2.12) is effectively equivalent to Eq.(2.11).

Now, we solve Eq.(2.11), i.e. $c_{0}+c_{1} z_{1}+c_{1}^{\prime} z_{3}+c_{3} z_{3} z_{1}^{2}=0, c_{0}+\left(c_{1}+c_{1}^{\prime}\right) z_{2}+c_{3} z_{2}^{3}=0$, and $c_{0}+c_{1} z_{3}+c_{1}^{\prime} z_{1}+c_{3} z_{1} z_{3}^{2}=0$. From the first and third equations, we obtain constraints on the coefficients $c_{0}, c_{1}, c_{1}^{\prime}$ and $c_{3}$,

$$
c_{1}-c_{1}^{\prime}=c_{3} z_{1} z_{3}, \quad c_{0}=-\left(c_{1}^{\prime}+c_{3} z_{1} z_{3}\right)\left(z_{1}+z_{3}\right) .
$$


By substituting Eqs.(2.13) into the second equation of (2.11), we obtain $c_{3}\left[z_{2}^{3}+z_{1} z_{3} z_{2}-z_{1} z_{3}\left(z_{1}+\right.\right.$ $\left.\left.z_{3}\right)\right]+c_{1}^{\prime}\left(2 z_{2}-z_{1}-z_{3}\right)=0$, i.e.

$$
c_{1}\left[z_{2}^{3}+z_{1} z_{3} z_{2}-z_{1} z_{3}\left(z_{1}+z_{3}\right)\right]-c_{1}^{\prime}\left(z_{2}^{2}-z_{1} z_{3}\right) z_{2}=0
$$

Note that Eq.(2.14) leads to the Sumino relation (1.5) in the limit of $c_{1}^{\prime} \rightarrow 0$. Since $c_{1}^{\prime} / c_{1}$ is given by

$$
\frac{c_{1}^{\prime}}{c_{1}}=\frac{\lambda_{2}^{\prime}}{\lambda_{2}} \frac{z_{2}^{2}+2 z_{1} z_{3}}{z_{1}^{2}+z_{2}^{2}+z_{3}^{2}}
$$

Eq.(2.14) is written as

$$
z_{2}^{3}+z_{1} z_{3} z_{2}-z_{1} z_{3}\left(z_{1}+z_{3}\right)-\rho \frac{\left(z_{2}^{2}+2 z_{1} z_{3}\right)\left(z_{2}^{2}-z_{1} z_{3}\right) z_{2}}{z_{1}^{2}+z_{2}^{2}+z_{3}^{2}}=0
$$

where $\rho=\lambda_{2}^{\prime} / \lambda_{2}$.

We can normalize the parameters $z_{i}$ as

$$
z_{1}+z_{2}+z_{3}=\sqrt{\frac{3}{2}}, \quad z_{1}^{2}+z_{2}^{2}+z_{3}^{2}=1+\xi
$$

without losing generality (by adjusting the value $v_{0}$ suitably). Then, the parameter $\xi$ corresponds to $\xi$ defined in Eq.(1.8). Since $z_{1}+z_{3}=\sqrt{3 / 2}-z_{2}$, and

$$
z_{1} z_{3}=\frac{1}{2}\left[\left(z_{1}+z_{3}\right)^{2}-\left(z_{1}^{2}+z_{3}^{2}\right)\right]=z_{2}^{2}-\sqrt{\frac{3}{2}} z_{2}+\frac{1}{4}(1-2 \xi)
$$

we can regard Eq.(2.16) as an equation for $z_{2}$, and we can solve it numerically. [The cubic equation (2.16) with $\rho=0$ (i.e. the Sumino equation) has only one real solution, while a general case with $\rho \neq 0$ has two real solutions. Therefore, of two numerical solutions, we will choose a solution which is nearly equal to the Sumino solution.] For typical values of $\rho, \rho=(0,1,2)$, we obtain $\kappa=(2.20867,2.1146,1.9792) \times 10^{-3}$ at $\xi=0$. Therefore, the case with $\rho \simeq 1-2$ is in favor of the observed value $\kappa^{\text {pole }}=(2.0633 \pm 0.001) \times 10^{-3}$, if we assume that the Sumino mechanism correctly works. However, at present, the parameter $\rho=\lambda_{2}^{\prime} / \lambda_{2}$ is free, so that we can predict neither values of $\kappa(\mu)$ or $\xi(\mu)$. In the next section, we will speculate about the ratio $\lambda_{2}^{\prime} / \lambda_{2}$

\section{Speculation}

In this section, we speculate about a value of $\lambda_{2}^{\prime} / \lambda_{2}$, and thereby, we speculate values of $\xi(\Lambda)$ and $\kappa(\Lambda)$.

In the previous section, we have considered that $\lambda_{2}$ and $\lambda_{2}^{\prime}$ terms come from $\operatorname{Tr}[A B C D] \rightarrow$ $\operatorname{Tr}[A B] \operatorname{Tr}[C D]$. Since $\operatorname{Tr}[A A B B]=\operatorname{Tr}[B A A B]=\operatorname{Tr}[B B A A]=\operatorname{Tr}[A B B A]$ and $\operatorname{Tr}[A B A B]=$ 
$\operatorname{Tr}[B A B A]$, we count the terms as $\operatorname{Tr}[A A B B] \rightarrow 2(\operatorname{Tr}[A A] \operatorname{Tr}[B B]+\operatorname{Tr}[A B] \operatorname{Tr}[A B])$ and $\operatorname{Tr}[A B A B] \rightarrow$ $2 \operatorname{Tr}[A B] \operatorname{Tr}[A B]$, respectively. Therefore, we consider

$$
\lambda_{1} \operatorname{Tr}[\Phi \Phi \tilde{\Phi} \tilde{\Phi}]+\lambda_{1}^{\prime} \operatorname{Tr}[\Phi \tilde{\Phi} \Phi \tilde{\Phi}] \rightarrow \lambda_{1} \operatorname{Tr}[\Phi \Phi] \operatorname{Tr}[\tilde{\Phi} \tilde{\Phi}]+\left(\lambda_{1}+\lambda_{1}^{\prime}\right) \operatorname{Tr}[\Phi \tilde{\Phi}] \operatorname{Tr}[\Phi \tilde{\Phi}],
$$

except for a common factor. On the other hand, independent arrangements of two $A$ and two $B$ are $A A B B, A B A B, A B B A, B B A A, B A B A$ and $B A A B$, so that we speculate $\lambda_{1}^{\prime} / \lambda_{1}=1 / 2$. Therefore, we obtain

$$
\rho \equiv \frac{\lambda_{2}^{\prime}}{\lambda_{2}}=\frac{\lambda_{1}+\lambda_{1}^{\prime}}{\lambda_{1}}=\frac{3}{2}
$$

The value $\rho=3 / 2$ predicts a value of $\kappa$

$$
\kappa(\xi=0)=2.0653 \times 10^{-3},
$$

which is in excellent agreement with the observed value $\kappa^{\text {pole }}=(2.0633 \pm 0.001) \times 10^{-3}$. However, as we have shown in Table 1, in the yukawaon model, the value of $\xi$ will be not zero, unless the Sumino mechanism exactly works.

In Fig.2, we illustrate a predicted $\xi(\mu)-\kappa(\mu)$ relation, where the predicted values $\kappa(\mu)$ are estimated for input values of $\xi(\mu)$ which correspond to the values of $\xi^{o b s}(\mu)$ at $\mu=m_{Z}, 10^{3}$ $\mathrm{GeV}, 10^{9} \mathrm{GeV}, 10^{12} \mathrm{GeV}$ and $2 \times 10^{16} \mathrm{GeV}$ in Table 1, respectively. As seen in Fig.2, the curve of $\xi(\mu)-\kappa(\mu)$ is crossed with the curve of $\xi(\mu)^{o b s}-\kappa^{o b s}(\mu)$ at $\mu \sim 10^{4} \mathrm{GeV}$. Of course, the crossed point depends on the value of $\tan \beta$. For a reference, in Fig.3, we also plot a $\xi-\kappa$ relation for the case with $\tan \beta=50$. The curves for $\rho=3 / 2$ and $\rho=1$ have crossing points with the $\xi^{o b s}-\kappa^{o b s}$ curve at $\mu \sim 10^{2} \mathrm{GeV}$ and $\mu \sim 10^{11} \mathrm{GeV}$, respectively. If we accept the speculated value $\rho=3 / 2$, we are obliged to consider $\Lambda \sim 10^{2} \mathrm{GeV}$, so that the case with $\tan \beta=50$ is unlikely. We consider that the value of $\Lambda$ is of the order of $10^{4} \mathrm{GeV}$ or more, so that the value of $\tan \beta$ must be of the order of 10 or less.

\section{VEV matrix forms $\langle E\rangle$ and $\langle\bar{T}\rangle$}

So far, we have not mentioned the origin of the VEV forms $\langle E\rangle$ and $\langle\bar{T}\rangle$. In Sec.2, we have a priori assumed their VEV forms (2.8). In a non-SUSY model, we can readily give an explicit scalar potential form which leads to the form $\langle E\rangle=v_{E} \mathbf{1}$. However, in general, in a SUSY model, it is hard to give a superpotential which leads to the form $\langle E\rangle=v_{E} \mathbf{1}$, although we can give $\langle E\rangle\langle\bar{E}\rangle \propto \mathbf{1}$. For example, we assume that a super potential

$$
W_{E}=\frac{\lambda_{1}^{E}}{\Lambda} \operatorname{Tr}[E \bar{E} E \bar{E}]+\frac{\lambda_{2}^{E}}{\Lambda} \operatorname{Tr}^{2}[E \bar{E}]
$$

where we have chosen $R$ charge parameters as $Q_{R}(E \bar{E})=r_{1}+2 r_{2}=1$. Supersymmetric vacuum conditions $\partial W_{E} / \partial E=0$ and $\partial W_{E} / \partial \bar{E}=0$ demand

$$
\lambda_{1}^{E} \bar{E} E+\lambda_{2}^{E} \operatorname{Tr}[\bar{E} E] \mathbf{1}=0,
$$

together with an additional condition for the coefficients $\lambda_{2}^{E} / \lambda_{1}^{E}=-1 / 3$. One way to obtain a solution $\langle E\rangle=v_{E} \mathbf{1}$ from $\bar{E} E \propto \mathbf{1}$ is to assume that our $\mathrm{U}(3)$ flavor symmetry is broken into $\mathrm{O}(3)$ 
above $\mu \sim \Lambda$ by $\langle E\rangle$, because we know that a $\mathrm{U}(3)$ symmetry is broken into an $\mathrm{O}(3)$ symmetry when a field $\mathbf{6}$ of $\mathrm{U}(3)$ takes a VEV form $\langle\mathbf{6}\rangle \propto \mathbf{1}$.

Another way is to assume that our U(3) is gauge symmetry according to Sumino's idea [2, 3]. Then, we can use a $D$-term condition for fields $\mathbf{6}$ and $\overline{\mathbf{6}}$ which are 6 -plet and $6^{*}$-plet of $\mathrm{U}(3)$, respectively:

$$
\sum_{\mathbf{6}} \operatorname{Tr}\left[\mathbf{6}^{\dagger} \lambda_{a} \mathbf{6}+\mathbf{6} \lambda_{a}^{T} \mathbf{6}^{\dagger}\right]-\sum_{\overline{\mathbf{6}}} \operatorname{Tr}\left[\overline{\mathbf{6}}^{\dagger} \lambda_{a} \overline{\mathbf{6}}+\overline{\mathbf{6}} \lambda_{a}^{T} \overline{\mathbf{6}}^{\dagger}\right]=0
$$

where $\lambda_{a}(a=1,2, \cdots, 8)$ are Gell-Mann's $\lambda$ matrices in $\mathrm{SU}(3)$ and $\lambda_{0}=\sqrt{2 / 3} 1$. (The $D$-term condition does not put any constraint on the fields $\Phi$ and $\tilde{\Phi}$ which are $\mathbf{8}+\mathbf{1}$ of U(3).) If we assume that $\langle\mathbf{6}\rangle$ and $\langle\overline{\mathbf{6}}\rangle$ are diagonal, the condition (4.3) is applied only to the cases $\lambda_{3}, \lambda_{8}$ and $\lambda_{0}$. A solution $E=\bar{E}=v_{E} \mathbf{1}$ under the constraint $E \bar{E} \propto \mathbf{1}$ satisfies a relation

$$
\operatorname{Tr}\left[E^{\dagger} \lambda_{a} E+E \lambda_{a}^{T} E^{\dagger}\right]-\operatorname{Tr}\left[\bar{E}^{\dagger} \lambda_{a} \bar{E}+\bar{E} \lambda_{a}^{T} \bar{E}^{\dagger}\right]=0
$$

Similarly, if we introduce a field $T$, which is $\mathbf{6}$ of $\mathrm{U}(3)$, in addition to $\bar{T}$, we can obtain $\langle T\rangle\langle\bar{T}\rangle \propto \mathbf{1}$. Considering $\langle T\rangle^{T}=\langle T\rangle$ and $\langle\bar{T}\rangle^{T}=\langle\bar{T}\rangle$, we take specific forms

$$
\langle T\rangle=\left(\begin{array}{ccc}
0 & 0 & v_{1} \\
0 & v_{2} & 0 \\
v_{1} & 0 & 0
\end{array}\right), \quad\langle\bar{T}\rangle=\left(\begin{array}{ccc}
0 & 0 & \bar{v}_{1} \\
0 & \bar{v}_{2} & 0 \\
\bar{v}_{1} & 0 & 0
\end{array}\right)
$$

of solutions for $\langle T\rangle\langle\bar{T}\rangle \propto \mathbf{1}$. Then, we can also see that a solution $\langle T\rangle=\langle\bar{T}\rangle$ with $v_{1}=v_{2}$ under the constraint $T \bar{T} \propto \mathbf{1}$ satisfies

$$
\operatorname{Tr}\left[T^{\dagger} \lambda_{a} T+T \lambda_{a}^{T} T^{\dagger}\right]-\operatorname{Tr}\left[\bar{T}^{\dagger} \lambda_{a} \bar{T}+\bar{T} \lambda_{a}^{T} \bar{T}^{\dagger}\right]=0
$$

Of the fields with $\mathbf{6}$ and $\overline{\mathbf{6}}$ of $\mathrm{U}(3)$ in the present model, for the last one $\Theta_{T}$, the VEV has to be $\left\langle\Theta_{T}\right\rangle=0$ from the $D$-term condition (4.3), because we already have relations (4.4) and (4.6) for $E, \bar{E}, T$ and $\bar{T}$. This solution $\left\langle\Theta_{T}\right\rangle=0$ is consistent with the requirement $\left\langle\Theta_{T}\right\rangle=0$ in Eq.(2.6).

\section{Concluding remarks}

In conclusion, on the basis of a yukawaon model, we have investigated the Sumino relation (1.2) for the charged lepton masses. As well as the Sumino model [3], where there is a $\lambda_{2}^{S}$ term in addition to the Sumino term (the $\lambda_{1}^{S}$ term) in the scalar potential (1.4), in the present model, too, we have additional terms, $c_{1}^{\prime}$ terms, in addition to the Sumino terms ( $c_{1}$-terms) in Eq.(2.14). In the Sumino model, since the contribution of the $\lambda_{2}^{S}$ term is considerably large compared with that of the $\lambda_{1}^{S}$ term for the realistic mass values, we must consider that $\lambda_{2}^{S} / \lambda_{1}^{S} \sim 10^{-2}$ in order to give the observed electron mass value. In other words, a predicted value of the electron mass is highly sensitive to the second term $\left(\lambda_{2}^{S}\right.$-term). On the other hand, in the present model, the $c_{1}^{\prime}$ terms less contribute to the essential terms ( $c_{1}$ terms), so that existence of the $c_{1}^{\prime}$ terms does not so spoil the Sumino equation (1.5) even if we take $\rho \sim 1$, and rather, the existence is in favor of the numerical fitting. 
We would like to emphasize that we have never used the charged lepton mass relation (1.1). Although we have put $K=(2 / 3)(1+\xi)$, we have never assumed that the parameter $\xi$ is small. Nevertheless, as shown in Figs.2 and 3, we can conclude that the values of $\xi(\Lambda)$ must be considerably small in order to accommodate the predicted $\xi-\kappa$ curves to the running mass values.

If we accept a speculation $\lambda_{2}^{\prime} / \lambda_{2}=3 / 2$, we obtain two options: One option is to consider that we accept the Sumino mechanism, so that the prediction (3.3) at $\xi=0$ is excellently in favor of the observed value (1.7) of $\kappa^{\text {pole }}$. Another option is to consider that the energy scale $\Lambda$ in the yukawaon model is $\Lambda \sim 10^{4} \mathrm{GeV}$ because the curve of the predicted value $\kappa(\mu)$ crosses with the curve of the observed value $\kappa(\mu)^{\text {obs }}$ at $\mu \sim 10^{4} \mathrm{GeV}$ as seen in Fig.2. (We have assumed that $\mathrm{U}(3)$ is not gauged, or that the contributions due to $\mathrm{U}(3)$ gauge bosons are negligibly small.) The latter scenario provides fruitful phenomenology in $\mathrm{TeV}$ region physics. Since in past yukawaon models the energy scale $\Lambda$ was taken as $\Lambda \sim 10^{14-16} \mathrm{GeV}$, all results which were obtained from the old yukawaon models must be re-considered.

In the present model, the VEV of the yukawaon $Y_{e}$ is given by $\left\langle Y_{e}\right\rangle \propto\langle\Phi\rangle\left\langle E_{e}\right\rangle\langle\Phi\rangle^{T}$ (exactly speaking, $\left\langle Y_{e}\right\rangle \propto \lambda_{Y 1}\langle\Phi\rangle\left\langle E_{e}\right\rangle\langle\Phi\rangle^{T}+\lambda_{Y 2}\left(\langle\Phi\rangle\langle\Phi\rangle\left\langle E_{e}\right\rangle+\left\langle E_{e}\right\rangle\langle\Phi\rangle^{T}\langle\Phi\rangle^{T}\right)$ ). Note that $\Phi$ (and $\bar{\Phi}$ ) have no sector charge, and only the filed $E_{e}$ has a non-zero sector charge $Q_{X}$. Therefore, we may consider a general form

$$
\left\langle Y_{f}\right\rangle \propto\langle\Phi\rangle\left\langle E_{f}\right\rangle\langle\Phi\rangle^{T}
$$

Since we have assumed that $\left\langle E_{e}\right\rangle$ is real, we could reduce $\operatorname{Tr}\left[Y_{e}\right]$ to $\operatorname{Tr}\left[D_{e}\right] \propto m_{e}+m_{\mu}+m_{\tau}\left(D_{f}\right.$ is a diagonalized matrix defined by $U_{f}^{T} Y_{f} U_{f}=D_{f}$ ), while, for a general yukawaon $Y_{f}$, which is 6 of $\mathrm{U}(3)$, i.e. which is not Hermitian, we cannot reduce $\operatorname{Tr}\left[Y_{f}\right]$ to such a form, because $U_{f} U_{f}^{T} \neq 1$. Therefore, only for the charged lepton sector, we can express the quantities $K$ and $\kappa$ by the ratios $\operatorname{Tr}\left[\Phi \Phi^{T}\right] / \operatorname{Tr}^{2}[\Phi]$ and $\operatorname{det}(\Phi) / \operatorname{Tr}^{3}[\Phi]$, respectively, while, for other sectors, we cannot obtain such relations (1.3) and (1.6). We think that this is a reason that $K_{f} \simeq 2 / 3$ are not satisfied [11] in other sectors (e.g. quark sectors).

As we have stated in Sec.1, although the present topic appears to be narrow and restricted, we think that the problems proposed by Sumino will provide a vital clue to new physics. If we take the speculation $\Lambda \sim 10^{4} \mathrm{GeV}$ seriously, we may expect possibility that the model provides fruitful and visible effects in $\mathrm{TeV}$ region physics. We hope that the present model can give some hints for fruitful developments of the Sumino model.

\section{Acknowledgments}

The author would like to thank Y. Sumino for valuable and helpful conversations (and also Particle Physics Group at Tohoku University for their hospitality). He also thanks S. Yamaguchi, T. Yamashita, Y. Hyakutake and N. Uekusa for helpful discussions and comments on a framework of a SUSY scenario. Especially, he is indebted to Yamashita for the idea of $D$-term condition. This work is supported by the Grant-in-Aid for Scientific Research (C), JSPS, (No.21540266). 


\section{References}

[1] Y. Koide, Lett. Nuovo Cimento 34 (1982) 201; Phys. Lett. B120 (1983) 161; Phys. Rev. D28 (1983) 252.

[2] Y. Sumino, Phys. Lett. B671 (2009) 477.

[3] Y. Sumino, JHEP 0905 (2009) 075.

[4] Y. Koide, Mod. Phys. Lett. A5 (1990) 2319.

[5] Y. Koide, Phys. Lett. B681 (2009) 68.

[6] Y. Koide, Phys. Rev. D78 (2008) 093006.

[7] Y. Koide, Phys. Rev. D79 (2009) 033009.

[8] Z.-z. Xing, H. Zhang and S. Zhou, Phys. Rev. D77 (2008) 113016.

[9] Y. Koide, Phys. Lett. B662 (2008) 43.

[10] Y. Koide, Phys. Lett. B665 (2008) 227.

[11] N. Li and B.-Q. Ma, Phys. Rev. D73 (2006) 01309; Z.-z. Xing and H. Zhang, Phys. Lett. B635 (2006) 107; Y. Koide, J. Phys. G 34 (2007) 1653. 


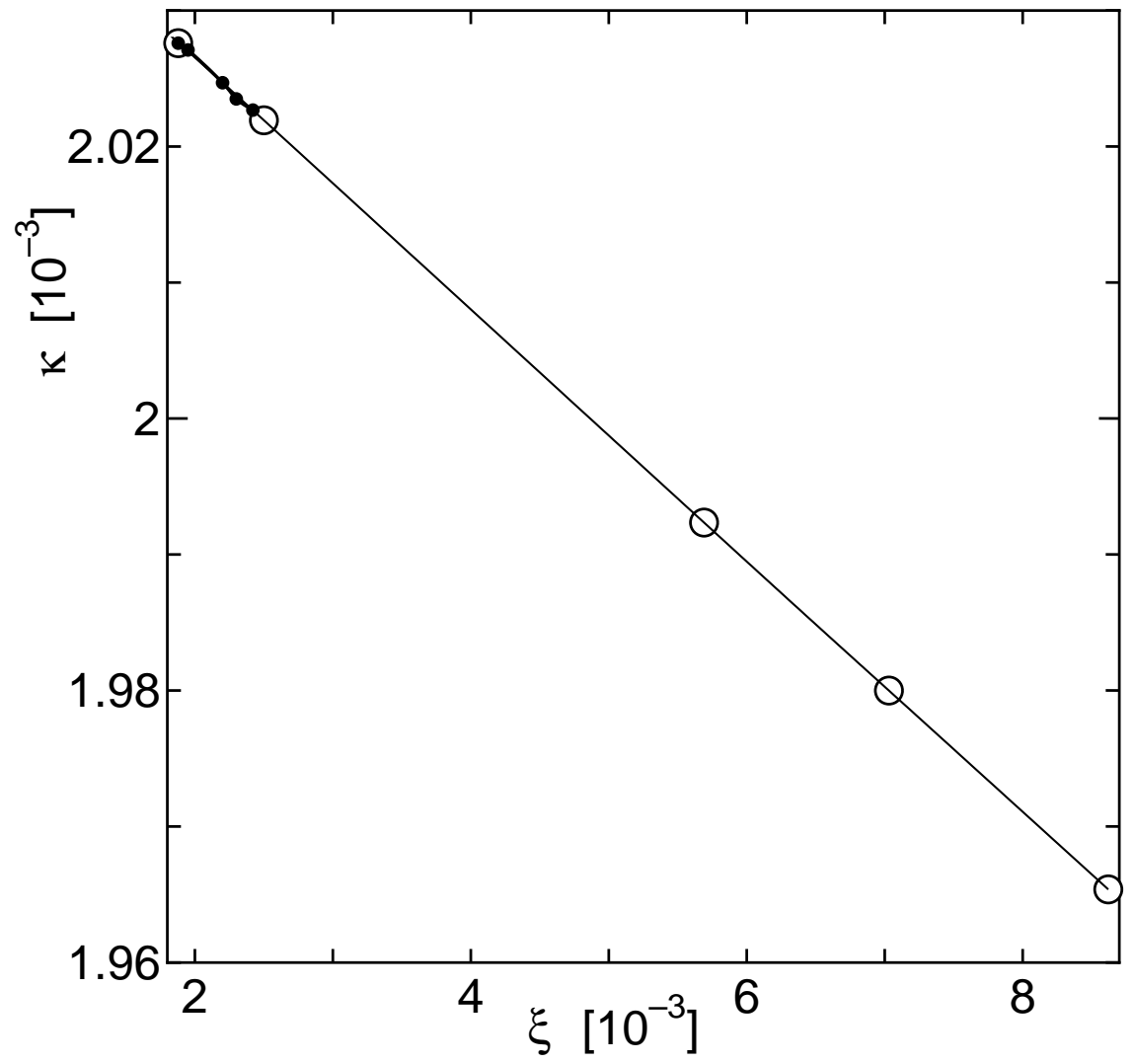

Fig. 1 Relation between $\xi(\mu)$ and $\kappa(\mu)$ estimated from running masses. One (a thick line with filled-in circle points) is for the case $\tan \beta=10$, another one (a thin line with opened circle points) is for $\tan \beta=50$. The data have been quoted from Xing et al. [8]. The data points from the left to the right correspond to $\xi-\kappa$ values at $\mu=m_{Z}, 10^{3} \mathrm{GeV}, 10^{9} \mathrm{GeV}, 10^{12} \mathrm{GeV}$ and $2 \times 10^{16} \mathrm{GeV}$, respectively. 


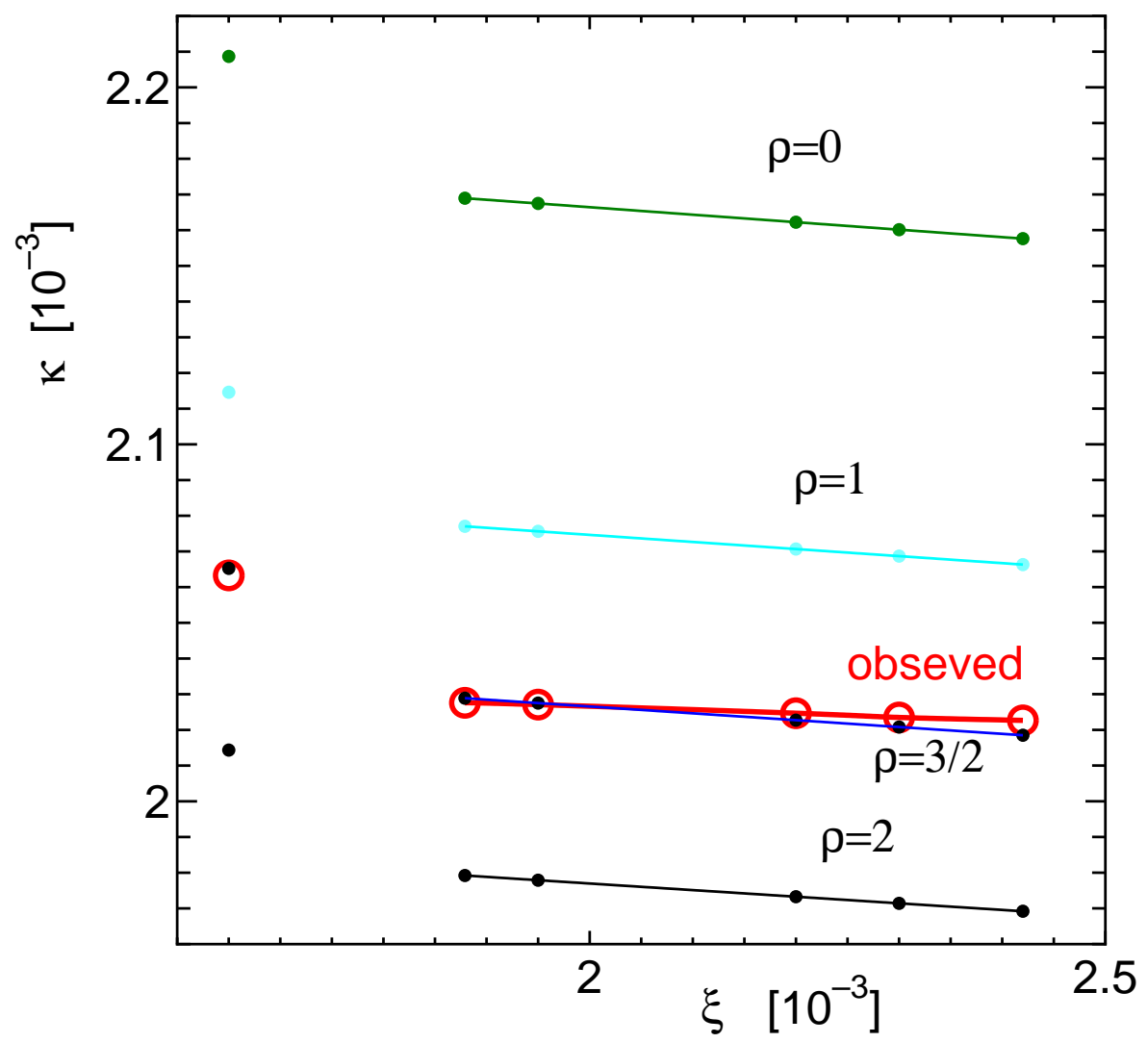

Fig. 2 Relation between $\xi(\mu)$ and $\kappa(\mu)$ for the case $\tan \beta=10$. A thick line with opened circles is plotted by values given in Table 1 . A curve for $\rho=0$ denotes the Sumino relation (1.2). Curves for $\rho=1,3 / 2$ and 2 are examples of predictions in the present model. The data points from the left to the right correspond to $\xi-\kappa$ values at $\mu=m_{Z}, 10^{3} \mathrm{GeV}, 10^{9} \mathrm{GeV}, 10^{12} \mathrm{GeV}$ and $2 \times 10^{16} \mathrm{GeV}$, respectively. On the left of those $\xi-\kappa$ curves, for a reference, values of $\kappa$ at $\xi=0$ are plotted together with observed value $\kappa^{\text {pole }}$. 


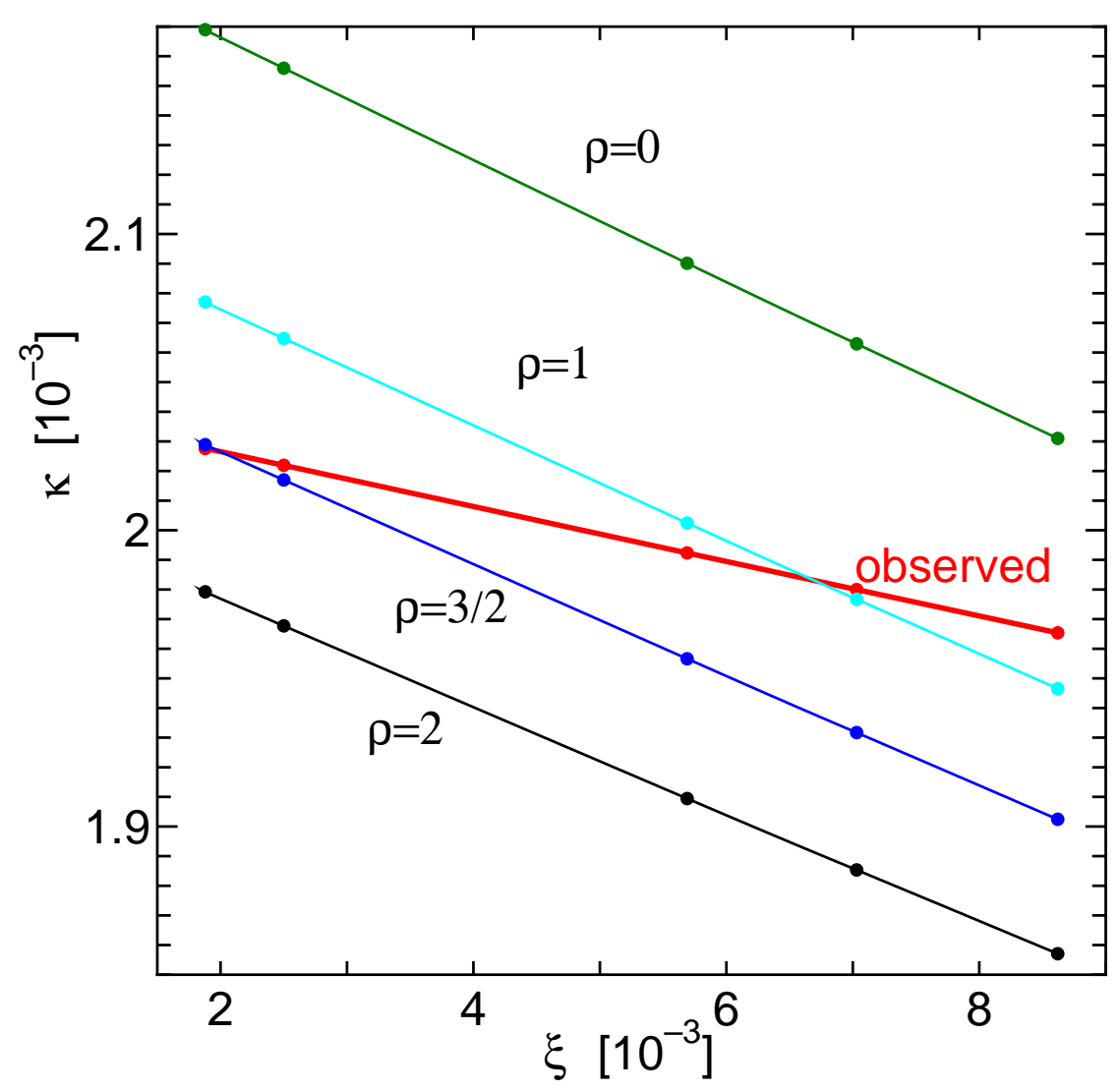

Fig. 3 Relation between $\xi(\mu)$ and $\kappa(\mu)$ for the case $\tan \beta=50$. The data points from the left to the right correspond to $\xi-\kappa$ values at $\mu=m_{Z}, 10^{3} \mathrm{GeV}, 10^{9} \mathrm{GeV}$, $10^{12} \mathrm{GeV}$ and $2 \times 10^{16} \mathrm{GeV}$, respectively. 